\title{
A Genetic-Algorithms-Based Approach for Programming Linear and Quadratic Optimization Problems with Uncertainty
}

\author{
Weihua Jin, Zhiying Hu, and Christine W. Chan \\ Energy Informatics Laboratory, Faculty of Engineering and Applied Science, University of Regina, \\ Regina, SK, Canada S4S 0A2 \\ Correspondence should be addressed to Christine W. Chan; christine.chan@uregina.ca
}

Received 18 June 2013; Accepted 22 October 2013

Academic Editor: Yudong Zhang

Copyright (C) 2013 Weihua Jin et al. This is an open access article distributed under the Creative Commons Attribution License, which permits unrestricted use, distribution, and reproduction in any medium, provided the original work is properly cited.

This paper proposes a genetic-algorithms-based approach as an all-purpose problem-solving method for operation programming problems under uncertainty. The proposed method was applied for management of a municipal solid waste treatment system. Compared to the traditional interactive binary analysis, this approach has fewer limitations and is able to reduce the complexity in solving the inexact linear programming problems and inexact quadratic programming problems. The implementation of this approach was performed using the Genetic Algorithm Solver of MATLAB (trademark of MathWorks). The paper explains the genetic-algorithms-based method and presents details on the computation procedures for each type of inexact operation programming problems. A comparison of the results generated by the proposed method based on genetic algorithms with those produced by the traditional interactive binary analysis method is also presented.

\section{Introduction}

Economic optimization in operation planning of municipal solid waste management was first proposed in the 1960s [1]. Since then, different models of waste management planning have been proposed, which include linear programming [25], mixed integer linear programming, dynamic programming, multiobjective programming [6-8], and hybrids of these methods combined with probability, fuzzy, and inexact analyses [9-12]. The objectives of these waste management models include reduction of total cost, protection of the environment, and reuse of waste material and energy.

In real-life engineering problems, the available information often cannot be represented as deterministic numbers or distribution functions. Instead, it is often possible to represent the available information as inexact numbers, which can be readily used in the inexact programming models. This may be due to the fact that decision makers often prefer to have an inexact representation of uncertainty than provide a specification for distributions of fuzzy sets [13-17]. For operational planning, the inexact analysis approach typically treats the uncertain parameters as intervals with known upper and lower bounds but unclear distributions. A major advantage of inexact analysis in operation planning is that variation of system performance and decision variables can be investigated by solving relatively simple submodels.

Research work on different kinds of inexact programming, such as inexact linear programming (ILP), inexact quadratic programming (IQP), inexact integer programming (IIP), inexact dynamic programming (IDP), and inexact multiobjective programming (IMOP) $[6,14,15,18-26]$, has been conducted. This paper presents an alternative heuristic-based method, which involves generic linear and quadratic programming with inexact information; the approach adopted involves the use of genetic algorithms (GA).

This paper is organized as follows. Section 2 presents the background of this research, which includes an introduction of the Genetic Algorithm Solver of MATLAB used for implementing the proposed method and the concepts of ILP, IQP, and their interactive binary analysis solution method [18]. Section 3 discusses the methodology of the proposed genetic-algorithms-based methods for solving inexact liner problems and inexact quadratic problems. Section 4 presents the solution of the IQP problem of solid waste disposal planning as a case study. 


\section{Background}

Linear programming and nonlinear programming are considered powerful optimization tools suitable for modeling and solving complex optimization problems in engineering. To handle uncertainty in real world data, inexact parameters and constraints are combined with various kinds of optimization techniques. Huang et al. [18-21] proposed two inexact nonlinear programming methods by introducing internal and fuzzy numbers into the quadratic programming (QP) frameworks. The methods of inexact quadratic programming (IQP) and inexact-fuzzy quadratic programming are applicable for operation planning of solid waste management systems. Often a detailed solution of IQP involves a large number of direct comparisons to interactively identify the uncertain relationships among the objective function and decision variables, whether the problems are mediumsized or larger-scaled. When these methods are applied to complicated and nonlinear problems, the number of direct comparisons can become exponential. In such a situation, we suggest that GA are a feasible problem-solving method.

GA have been applied as the optimization techniques for solving complex and nonlinear problems in operations research, industrial engineering, and management science. The GA method is a suitable optimization tool especially for solving problems, which involve nonsmooth and multimodal search spaces. An engineering problem that has traditionally been solved as an IQP problem often involves a large and uneven search space, for which a global optimal solution is often not required. Instead, we suggest that the GA-based method is a more effective problem-solving approach than some traditional inexact programming methods.

2.1. Genetic Algorithm Solver for MATLAB. For implementation of genetic algorithms, the Genetic Algorithm Solver of Global Optimization Toolbox (GASGOT), developed by MATLAB (Trademark of MathWorks), has been adopted. GASGOT implements simulated evolution in the MATLAB environment using both binary and floating point representations and ordered base representation. This enables flexible implementation of the genetic operators, selection functions, termination functions, and evaluation functions. GASGOT was developed by the Department of Industrial Engineering of North Carolina State University as a toolbox of MATLAB. Hence, it runs in a MATLAB workspace and can be easily invoked by other programs. GASGOT supports implementation of binary chromosomes, binary mutation, and simple crossover. For floating point representation, the operators of uniform mutation, nonuniform mutation, multinonuniform mutation, boundary mutation, simple crossover, arithmetic crossover, and heuristic crossover are defined.

The GASGOT is adopted as the problem-solving engine of both the GA linear program and GA nonlinear program; all the applications and numeric examples were calculated using this solver in MATLAB.

2.2. Inexact Linear Programming and Its Problem-Solving Approach. To support decision making involving uncertainties, Huang et al. [20, 22] proposed an interactive binary analysis to solve the inexact linear programming (ILP) problem.

A typical ILP problem can be expressed as follows:

$$
\begin{array}{ll}
\operatorname{Max} & f^{ \pm}=\sum_{j=1}^{n}\left[c_{j}^{ \pm} x_{j}^{ \pm}\right], \\
\text {s.t. } & \sum_{j=1}^{n} a_{i j}^{ \pm} x_{j}^{ \pm} \leq b_{i}^{ \pm}, \quad i=1,2, \ldots m, \\
& x_{j}^{ \pm} \geq 0, \quad j=1,2, \ldots, n,
\end{array}
$$

where $a_{i j}^{ \pm}, b_{i}^{ \pm}$, and $c_{j}^{ \pm}$are inexact parameters and $x_{j}^{ \pm}$is an inexact variable. It is assumed that an optimal solution exists. For an inexact number $g^{ \pm} \in\left[g^{-}, g^{+}\right], g^{+}$and $g^{-}$are the upper and lower bounds, respectively. follows.

The traditional binary solution procedure is specified as

Step 1. Group symbols for inexact coefficients $c_{j}^{ \pm}$; let former $k_{1}$ coefficients be positive and latter $k_{2}$ be negative; $k_{1}+k_{2}=$ $n$.

Step 2. Define the upper and lower bounds of the objective function as $f^{+}$and $f^{-}$.

Step 3. Define absolute values and signs for the coefficients of the constraints $a_{i j}^{ \pm}$.

Step 4. Define the relationships between the decision variables $x_{j}^{ \pm}$and the absolute value of the coefficients of the constraints $\left|a_{i j}^{ \pm}\right|$.

Step 5. Formulate constraints corresponding to the upper and lower bounds of the objective function $f^{+}$and $f^{-}$.

Step 6. When the right-hand side of the constraints $b_{i}$ are also inexact numbers, define the relationships between $f^{ \pm}$and $b_{i}^{ \pm}$.

Step 7. Specify the two submodels.

For a detailed description of the procedure, see Huang et al. $[20,22,23]$.

2.3. Inexact Quadratic Programming and Its Problem-Solving Approach. A typical IQP problem is formulated as follows:

$$
\begin{aligned}
& \operatorname{Max} f^{ \pm}=\sum_{j=1}^{n}\left[c_{j}^{ \pm} x_{j}^{ \pm}+d_{j}^{ \pm}\left(x_{j}^{ \pm}\right)^{2}\right], \\
& \text { s.t. } \quad \sum_{j=1}^{n} a_{i j}^{ \pm} x_{j}^{ \pm} \leq b_{i}^{ \pm}, \quad i=1,2, \ldots m, \\
& x_{j}^{ \pm} \geq 0, \quad j=1,2, \ldots, n,
\end{aligned}
$$

where $a_{i j}^{ \pm}, b_{i j}^{ \pm}, c_{i j}^{ \pm}$, and $d_{i j}^{ \pm}$are inexact parameters, $x_{j}^{ \pm}$is an inexact variable, and it is assumed that an optimal solution exists. 
The solution procedure is similar to that of ILP but involves more complexity and computation; the main steps of the solution procedure are listed as follows; for a detailed description, see Huang et al. [18, 24, 25].

Step 1. Group symbols for inexact coefficients $c_{j}^{ \pm}$and $d_{i j}^{ \pm}$; when $c_{j}^{ \pm}$and $d_{i j}^{ \pm}$have the same signs, similar to the ILP, let former $k_{1}$ coefficients be positive and latter $k_{2}$ be negative; $k_{1}+k_{2}=n$. When $c_{j}^{ \pm}$and $d_{i j}^{ \pm}$have different signs, $2^{n}$ combinations of the upper and lower bounds of $x_{j}^{ \pm}$have to be formulated for the objective function, which will require a large number of computations.

Step 2. Define the upper and lower bounds of the objective function as $f^{+}$and $f^{-}$.

Step 3. Define the absolute values and signs for the coefficients of the constraints $a_{i j}^{ \pm}$and the relationships between the decision variables $x_{j}^{ \pm}$and the absolute value of the coefficients of the constraints $\left|a_{i j}^{ \pm}\right|$.

When some $x_{j}^{-}$corresponds to $f^{+}$and some $x_{j}^{+}$corresponds to $f^{-}$, the specification of the constraints $a_{i j}^{ \pm} x_{j}^{ \pm} \leq$ $b_{i}^{ \pm}$requires a comparison of the contribution of $x_{j}^{+}$and $x_{j}^{-}$ groups to the sum $\sum_{j=1}^{n} a_{i j}^{ \pm} x_{j}^{ \pm}$, when $f^{+}$is desired. When the problem is complex, a direct comparison of the dominance of $x_{j}^{+}$and $x_{j}^{-}$becomes impossible; then some simplification and assumption need to be considered, which will affect the quality of the result.

Step 4. Formulate the constraints corresponding to the upper and lower bounds of the objective function $f^{+}$and $f^{-}$.

Step 5. When the right-hand side of the constraints $b_{i}$ are also inexact numbers, define the relationships between $f^{ \pm}$and $b_{i}^{ \pm}$.

Step 6. Specify the two submodels.

The genetic-algorithms-based methods to solve the above inexact linear problem and inexact quadratic problem will be presented in the next section, and the results from the GAbased methods will be compared to those generated using the traditional approach in [18, 20, 22-25].

\section{Methodology}

3.1. Genetic-Algorithms-Based Method for Solution of ILP Problems (GAILP). A GA, as a heuristic search algorithm, has been adopted for solving the aforementioned ILP problem. In the GA approach, the upper and lower bounds of the inexact numbers of coefficients $a_{i j}^{ \pm}, b_{i}^{ \pm}$, and $c_{j}^{ \pm}$can be determined by substituting the initial suboptimal decision variables into the objective function. $f^{+}$and $f^{-}$can be calculated directly without any uncertainty in the coefficients. This approach is called the genetic-algorithms-based method for solving ILP problems or the GAILP method.

GAILP has been designed to include three stages, which are discussed as follows.
The objective of the first stage is to get an initial suboptimal $x_{j}^{s}$ for the following problem, which is transformed from the ILP problem defined in (1):

$$
\begin{aligned}
\operatorname{Max} & f^{ \pm}=\sum_{j=1}^{n}\left[c_{j}^{r} x_{j}^{s}\right], \\
\text { s.t. } \quad & \sum_{j=1}^{n} a_{i j}^{r} x_{j}^{s} \leq b_{i}^{r}, \quad i=1,2, \ldots, m, \\
& x_{j} \geq 0, \quad j=1,2, \ldots, n,
\end{aligned}
$$

where $a_{i j}^{r}, b_{i}^{r}$, and $c_{j}^{r}$ are random numbers that satisfy the continuous uniform distribution in the intervals of $\left[a_{i j}^{-}, a_{i j}^{+}\right]$, $\left[b_{i}^{-}, b_{i}^{+}\right]$, and $\left[c_{j}^{-}, c_{j}^{+}\right]$, respectively. Then, the problem is solved by the GA linear program solving engine of GASGOT, which uses the objective function in (3) as the positive term of the fitness function and the constraints of (1) as the negative punishment terms. Thus, a suboptimal solution $f^{s}$ can be identified and the corresponding decision variables of $x_{j}^{s}$ are also obtained.

In the second stage, the inexact coefficients of $a_{i j}^{ \pm}, b_{i}^{ \pm}$, and $c_{j}^{ \pm}$will be determined. Let the determined coefficients corresponding to $f^{+}$be $a_{i j}^{ \pm+}, b_{i}^{ \pm+}$, and $c_{j}^{ \pm+}$and those corresponding to $f^{-}$be $a_{i j}^{ \pm^{-}}, b_{i}^{ \pm^{-}}$, and $c_{j}^{ \pm^{-}}$. These two sets of coefficients can be obtained using the following method.

Substituting $x_{j}^{s}$ into (1) will convert (1) into the following equation:

$$
\begin{aligned}
\operatorname{Max} & f^{ \pm}=\sum_{j=1}^{n}\left[c_{j}^{ \pm} x_{j}^{s}\right], \\
\text { s.t. } \quad & \sum_{j=1}^{n} a_{i j}^{ \pm} x_{j}^{s} \leq b_{i}^{ \pm}, \quad i=1,2, \ldots, m, \\
& x_{j}^{s} \geq 0, \quad j=1,2, \ldots, n .
\end{aligned}
$$

To identify the coefficients $a_{i j}^{ \pm}, b_{i}^{ \pm}$, and $c_{j}^{ \pm}$corresponding to $f^{ \pm}$, a set of objective functions needs to be constructed and solved. Since $x_{j}^{s}$ are suboptimal variables, which tend to make the objective function closer to $f^{+}$, consider $a_{i j}^{ \pm}, b_{i}^{ \pm}$, and $c_{j}^{ \pm}$as variables; then the objective function of (5) can be constructed so as to find $c_{j}^{ \pm^{+}}$:

$$
\begin{array}{ll}
\operatorname{Max} & f^{ \pm}=\sum_{j=1}^{n}\left[c_{j}^{ \pm} x_{j}^{s}\right], \\
\text { s.t. } & \sum_{j=1}^{n} a_{i j}^{ \pm} x_{j}^{s} \leq b_{i}^{ \pm}, \quad i=1,2, \ldots, m .
\end{array}
$$

The coefficients $c_{j}^{ \pm^{+}}$are considered as corresponding to $f^{+}$. 
At the same time, the objective function presented in (6) can be constructed so as to find $c_{j}^{ \pm^{-}}$:

$$
\begin{aligned}
& \text { Min } \quad f^{ \pm}=\sum_{j=1}^{n}\left[c_{j}^{ \pm} x_{j}^{s}\right], \\
& \text { s.t. } \quad \sum_{j=1}^{n} a_{i j}^{ \pm} x_{j}^{s} \leq b_{i}^{ \pm}, \quad i=1,2, \ldots, m .
\end{aligned}
$$

There are two kinds of decision schemes for inexact programming problems, which are the conservative schemes and optimistic schemes [26]. The former assumes less risk than the latter, so that, for a maximization objective function, planning for the lower bound of an objective value represents the conservative scheme, and planning for the upper bound of an objective value represents the optimistic scheme [26]. In terms of constraints, the conservative scheme involves more rigorous or stringent constraints, and the optimistic scheme adopts more tolerant ones.

Thus, the problem of searching for $a_{i j}^{ \pm^{+}}$and $b_{i}^{ \pm^{+}}$of the optimistic scheme and corresponding to the upper bound of the objective value of $f^{+}$can be represented as follows:

$$
\begin{array}{ll}
\operatorname{Max} & \sum_{j=1}^{n} \mathrm{abs}\left(a_{i j}^{ \pm} x_{j}^{s}-b_{i}^{ \pm}\right), \\
\text {s.t. } & \sum_{j=1}^{n} a_{i j}^{ \pm} x_{j}^{s} \leq b_{i}^{ \pm}, \quad i=1,2, \ldots, m .
\end{array}
$$

The problem

$$
\begin{array}{ll}
\operatorname{Min} & \sum_{j=1}^{n} a b s\left(a_{i j}^{ \pm} x_{j}^{s}-b_{i}^{ \pm}\right), \\
\text {s.t. } & \sum_{j=1}^{n} a_{i j}^{ \pm} x_{j}^{s} \leq b_{i}^{ \pm}, \quad i=1,2, \ldots, m,
\end{array}
$$

will give $a_{i j}^{ \pm^{-}}$and $b_{i}^{ \pm^{-}}$of the conservative scheme, corresponding to the lower bound of the objective value of $f^{-}$.

Hence, the values of $a_{i j}^{ \pm^{+}}, b_{i}^{ \pm^{+}}$, and $c_{j}^{ \pm^{+}}$and $a_{i j}^{ \pm^{-}}, b_{i}^{ \pm^{-}}$, and $c_{j}^{ \pm-}$can be calculated.

In the third stage, the problem represented in (1) is converted into the following two subproblems.

For $f^{+}$,

$$
\begin{aligned}
& \operatorname{Max} f^{+}=\sum_{j=1}^{n}\left[c_{j}^{ \pm^{+}} x_{j}^{ \pm}\right], \\
& \text {s.t. } \quad \sum_{j=1}^{n} a_{i j}^{ \pm^{+}} x_{j}^{ \pm} \leq b_{i}^{ \pm^{+}}, \quad i=1,2, \ldots, m, \\
& x_{j}^{ \pm} \geq 0, \quad j=1,2, \ldots, n .
\end{aligned}
$$

For $f^{-}$,

$$
\begin{aligned}
\operatorname{Max} & f^{-}=\sum_{j=1}^{n}\left[c_{j}^{ \pm-} x_{j}^{ \pm}\right], \\
\text {s.t. } \quad & \sum_{j=1}^{n} a_{i j}^{ \pm-} x_{j}^{ \pm} \leq b_{i}^{ \pm-}, \quad i=1,2, \ldots, m, \\
& x_{j}^{ \pm} \geq 0, \quad j=1,2, \ldots, n .
\end{aligned}
$$

This step eliminates the inexact parameters in (1) and generates instead (9) and (10) as typical linear programming (LP) problems, which can be solved easily using the traditional methods. Generally speaking, the interactive binary algorithm of Huang et al. [20, 22] can be used for solving inexact linear problems reliably and relatively quickly for many real-life decision-making scenarios in the engineering field. However, this binary algorithm has some limitations. One of them, for example, is the limitation that the upper and lower bounds of an inexact coefficient cannot have different signs. By contrast, the GAILP does not have this kind of limitation because the GA method does not depend on any assumed distribution of the inexact parameter.

The following inexact linear problem demonstrates how GAILP is able to handle this situation:

$$
\begin{array}{ll}
\text { Max } & f^{ \pm}=[2,6] x_{1}^{ \pm}+[7.8,10.2] x_{2}^{ \pm}, \\
\text {s.t. } & x_{1}^{ \pm}+[-0.8,1.2] x_{2}^{ \pm} \leq 4.6, \\
& {[-6,-8.5] x_{1}^{ \pm}+12 x_{2}^{ \pm} \leq[-2,3],} \\
& x_{1}^{ \pm} \geq 0, \quad x_{2}^{ \pm} \geq 0 .
\end{array}
$$

In stage one, the suboptimal $f^{s}$ and the corresponding $x_{1}^{s}$ and $x_{2}^{s}$ are calculated:

$$
f^{s}=112, \quad x_{1}^{s}=10.1, \quad x_{2}^{s}=5.8 .
$$

In stage two, substituting $x_{1}^{s}$ and $x_{2}^{s}$ into (11), the two sets of problems for determining $a_{i j}^{ \pm^{+}}, b_{i}^{ \pm^{+}}$, and $c_{j}^{ \pm^{+}}$and $a_{i j}^{ \pm^{-}}, b_{i}^{ \pm^{-}}$, and $c_{j}^{ \pm^{-}}$are constructed for $f^{+}$and $f^{-}$, respectively.

For $f^{+}, c_{j}^{ \pm^{+}}$can be determined by solving the following problem:

$$
\begin{array}{ll}
\operatorname{Max} & f^{+}=c_{1}^{ \pm+} 10.1+c_{2}^{ \pm+} 5.8, \\
\text { s.t. } & 10.1+[-0.8,1.2] 5.8 \leq 4.6, \\
& {[-6,-8.5] 10.1+125.8 \leq[-2,3],} \\
& c_{1}^{ \pm^{+}} \leq[2,6], \quad c_{2}^{ \pm+} \leq[7.8,10.2] .
\end{array}
$$


And $a_{i j}^{ \pm^{+}}$and $b_{i}^{ \pm^{+}}$can be determined by solving the following problem:

$$
\begin{gathered}
\operatorname{Max} 4.6-\left(x_{1}^{s}+a_{12} x_{2}^{s}\right), \\
\operatorname{Max} b_{2}-\left(a_{21} x_{1}^{s}+12 x_{2}^{s}\right), \\
a_{12}=[-0.8,1.2], \\
a_{21}=[-6,-8.5], \\
b_{2}=[-2,3] .
\end{gathered}
$$

For $f^{-}, c_{j}^{ \pm^{-}}$can be determined by solving the following problem:

$$
\begin{array}{ll}
\text { Min } & f^{-}=c_{1}^{ \pm^{-}} 10.1+c_{2}^{ \pm^{-}} 5.8, \\
\text { s.t. } & 10.1+[-0.8,1.2] 5.8 \leq 4.6, \\
& {[-6,-8.5] 10.1+125.8 \leq[-2,3],} \\
& c_{1}^{ \pm^{-}} \leq[2,6], \quad c_{2}^{ \pm^{-}} \leq[7.8,10.2] .
\end{array}
$$

And $a_{i j}^{ \pm^{-}}$and $b_{i}^{ \pm^{-}}$can be determined by solving the following problem:

$$
\begin{gathered}
\operatorname{Min} 4.6-\left(x_{1}^{s}+a_{12} x_{2}^{s}\right), \\
\operatorname{Min} b_{2}-\left(a_{21} x_{1}^{s}+12 x_{2}^{s}\right), \\
a_{12}=[-0.8,1.2], \\
a_{21}=[-8.5,-6], \\
b_{2}=[-2,3] .
\end{gathered}
$$

By solving (13), (14), (15), and (16), the coefficients are calculated as follows:

$$
\begin{gathered}
a_{12}^{ \pm^{+}}=-0.8, \quad a_{21}^{ \pm^{+}}=-8.5, \quad b_{2}^{ \pm^{+}}=3, \\
c_{1}^{ \pm^{+}}=6, \quad c_{2}^{ \pm^{+}}=10.2, \\
a_{12}^{ \pm^{-}}=1.2, \quad a_{21}^{ \pm^{-}}=-6, \quad b_{2}^{ \pm^{-}}=-2, \\
c_{1}^{ \pm^{-}}=2, \quad c_{2}^{ \pm^{-}}=7.8 .
\end{gathered}
$$

In stage three, the two submodels for the optimistic scheme and the conservative scheme are constructed, respectively, as follows.

For the optimistic scheme, $f^{+}$,

$$
\begin{array}{ll}
\operatorname{Max} & f^{+}=6 x_{1}^{+}+10.2 x_{2}^{+}, \\
\text {s.t. } & x_{1}^{+}-0.8 x_{2}^{+} \leq 4.6, \\
& -8.5 x_{1}^{+}+12 x_{2}^{+} \leq 3, \\
& x_{1}^{+} \geq 0, \quad x_{2}^{+} \geq 0 .
\end{array}
$$

For the conservative scheme, $f^{-}$,

$$
\begin{array}{ll}
\operatorname{Max} & f^{-}=2 x_{1}^{-}+7.8 x_{2}^{-}, \\
\text {s.t. } & x_{1}^{-}+1.2 x_{2}^{-} \leq 4.6, \\
& -6 x_{1}^{-}+12 x_{2}^{-} \leq 2, \\
& x_{1}^{-} \geq 0, \quad x_{2}^{-} \geq 0 .
\end{array}
$$

The final result can be found by solving the previous two submodels:

$$
\begin{gathered}
f^{+}=149.04, \quad x_{1}^{+}=11.08, \quad x_{2}^{+}=8.1, \\
f^{-}=16.4, \quad x_{1}^{+}=3, \quad x_{2}^{+}=1.33 .
\end{gathered}
$$

From the previous calculation, it can be seen that the GAILP method can be used without any assumption of the upper and lower bounds of the inexact coefficients. In fact, this method effectively extends the scope of problems solvable using the methods of the inexact linear programming problem. Therefore, the GAILP method is more adaptable for real world applications of optimization problems with uncertainty. In the next section, this method will be extended to solve the inexact quadratic problems.

A sample inexact linear programming problem in [22] is as follows:

$$
\begin{array}{ll}
\operatorname{Max} & f^{ \pm}=c_{1} x_{1}^{ \pm}+c_{2} x_{2}^{ \pm}, \\
\text {s.t. } & a_{11} x_{1}^{ \pm}+a_{12} x_{2}^{ \pm} \leq b_{1}, \\
& a_{21} x_{1}^{ \pm}+a_{22} x_{2}^{ \pm} \leq b_{2},
\end{array}
$$

where $c_{1}=[26,30], \quad c_{2}=[-6,-5.5]$,

$$
\begin{gathered}
a_{11}=[8,10], \quad a_{12}=[-14,-12], \quad b_{1}=[3.8,4.2], \\
a_{21}=[2.4,2.8], \quad a_{22}=[3.4,4], \quad b_{2}=6.5 .
\end{gathered}
$$

By using the traditional interactive binary algorithm, two submodels are obtained:

$$
\begin{array}{ll}
\operatorname{Max} & f^{+}=30 x_{1}^{+}-5.5 x_{2}^{-}, \\
\text {s.t. } & 8 x_{1}^{+}-14 x_{2}^{-} \leq 4.2, \\
& 2.4 x_{1}^{+}+4 x_{2}^{-} \leq 6.5, \\
& x_{1}^{+} \geq 0, \quad x_{2}^{-} \geq 0, \\
& f^{-}=26 x_{1}^{-}-6.0 x_{2}^{+}, \\
\text {s.t. } & 10 x_{1}^{-}-12 x_{2}^{+} \leq 3.8, \\
& 2.8 x_{1}^{-}+3.4 x_{2}^{+} \leq 6.5, \\
& x_{1}^{-} \geq 0, \quad x_{2}^{+} \geq 0 .
\end{array}
$$

The result was $f^{+}=45.78, x_{1}=1.64$, and $x_{2}=0.64 ; f^{-}=$ $30.77, x_{1}=1.37$, and $x_{2}=0.79$. 


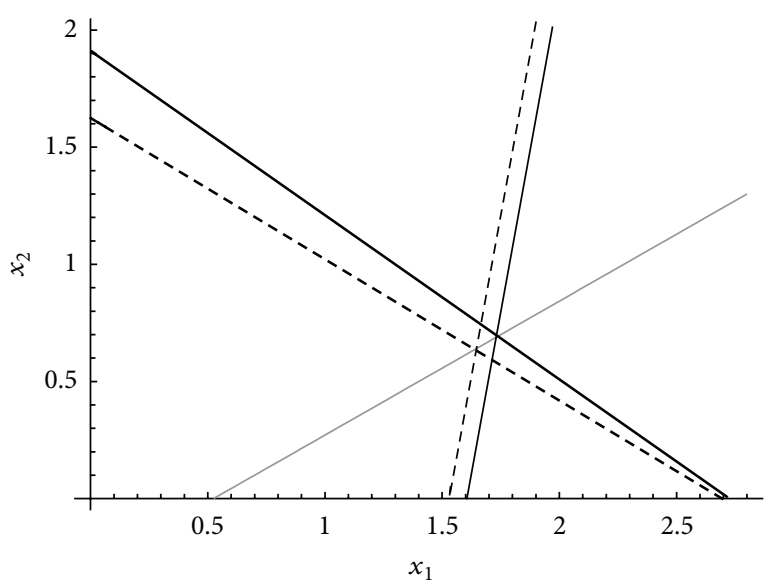

Figure 1: Optimistic scheme, $f^{+}$.

For a detailed description of the problem, see [22].

By using the GAILP method as stated in (21), the result can be calculated with the following objective functions:

$$
\begin{aligned}
& \operatorname{Max} f^{+}=30 x_{1}^{+}-5.5 x_{2}^{+}, \\
& \text {s.t. } 8 x_{1}^{+}-14 x_{2}^{+} \leq 4.2 \text {, } \\
& 2.4 x_{1}^{+}+3.4 x_{2}^{+} \leq 6.5 \text {, } \\
& x_{1}^{+} \geq 0, \quad x_{2}^{+} \geq 0 \text {, } \\
& \operatorname{Max} f^{-}=26 x_{1}^{-}-6.0 x_{2}^{-} \text {, } \\
& \text { s.t. } 10 x_{1}^{-}-12 x_{2}^{-} \leq 3.8 \text {, } \\
& 2.8 x_{1}^{-}+4 x_{2}^{-} \leq 6.5 \text {, } \\
& x_{1}^{-} \geq 0, \quad x_{2}^{-} \geq 0 \text {. }
\end{aligned}
$$

The result was $f^{+}=48.15, x_{1}=1.73$, and $x_{2}=0.69 ; f^{-}=$ 29.15, $x_{1}=1.29$, and $x_{2}=0.72$.

The GAILP method generates a solution, which is different from that obtained using the interactive binary analysis proposed in [22]. A comparison of the results will be discussed as follows.

For the $f^{+}$optimistic scheme, the GAILP method can generate a result that is guaranteed to be as close as possible to the upper bound of the constraints. Hence, the maximized value of the objective function is greater than that produced by the interactive binary analysis. For the $f^{-}$conservative scheme, the GAILP method has a higher probability of satisfying the requirements of the constraints as close as possible to the lowest limit. Hence, the maximized objective value is smaller.

In Figures 1, 2, 3, and 4 the bold lines denote the boundaries of the constraints, which limit the possible values for $x_{1}$ and $x_{2}$ to the left lower area. The constraint $a_{11} x_{1}^{ \pm}+a_{12} x_{2}^{ \pm} \leq b_{1}$ is shown in these figures as the grey bold solid lines, which is the same for both the interactive binary analysis and the GAILP method. The dark bold dotted lines represent the constraint of $a_{21} x_{1}^{ \pm}+a_{22} x_{2}^{ \pm} \leq b_{2}$ given by the interactive
TABLE 1: Legend for Figures 1 to 4.

The constraint $a_{21} x_{1}^{ \pm}+a_{22} x_{2}^{ \pm} \leq b_{2}$ given by interactive binary analysis

The constraint $a_{21} x_{1}^{ \pm}+a_{22} x_{2}^{ \pm} \leq b_{2}$ given by GAILP

The constraint $a_{11} x_{1}^{ \pm}+a_{12} x_{2}^{ \pm} \leq b_{1}$

Objective function line given by interactive binary analysis

Objective function line given by GAILP

binary analysis, and the dark bold solid lines represent the same constraint given by the proposed GAILP method.

The boundaries, together with the $x_{1}$ and $x_{2}$ axes, enclose the entire area defined by the constraints. The objective functions $f^{+}=30 x_{1}^{+}-5.5 x_{2}^{+}$or $f^{-}=26 x_{1}^{-}-6 x_{2}^{-}$are groups of parallel lines, as shown in the figures by the thin solid and dotted lines. According to different values of $x_{1}$ and $x_{2}$, these objective function lines would have different intercepts on both axes. These constraints restrict the objective function lines to cross with the constraints area, so that, at some vertex, the objective function would reach its extreme (i.e., maximized or minimized) values.

In Figures 1, 2, 3, and 4, the thin dotted lines are given by the interactive binary analysis, and the thin solid lines represent the objective functions given by the proposed GAILP method. The legend for Figure 1 to Figure 4 is shown in Table 1.

3.2. Genetic-Algorithms-Based Method for Solving IQP Problems (GAIQP). The GAILP method can be extended to solve the inexact quadratic programming (IQP) problems or other more complicated inexact nonlinear programming problems. The typical IQP problem was presented in Section 2 as (2).

In stage one, to obtain an initial suboptimal $x_{j}^{s}$ from a problem transformed from the IQP problem we use the following:

$$
\begin{array}{ll}
\operatorname{Max} & f=\sum_{j=1}^{n}\left[c_{j}^{r} x_{j}+d_{j}^{r}\left(x_{j}\right)^{2}\right], \\
\text { s.t. } & \sum_{j=1}^{n} a_{i j}^{r} x_{j}^{r} \leq b_{j}^{r}, \quad i=1,2, \ldots, m, \\
& x_{j} \geq 0, \quad j=1,2, \ldots, n,
\end{array}
$$

where $a_{i j}^{r}, b_{i}^{r}, c_{j}^{r}$, and $d_{j}^{r}$ are random numbers that satisfy the continuous uniform distribution in the intervals $\left[a_{i j}^{-}, a_{i j}^{+}\right]$, $\left[b_{i}^{-}, b_{i}^{+}\right],\left[c_{j}^{-}, c_{j}^{+}\right]$, and $\left[d_{j}^{-}, d_{j}^{+}\right]$. Then, a suboptimal solution $f^{s}$ can be identified, and the corresponding decision variables $x_{j}^{s}$ are also obtained. 


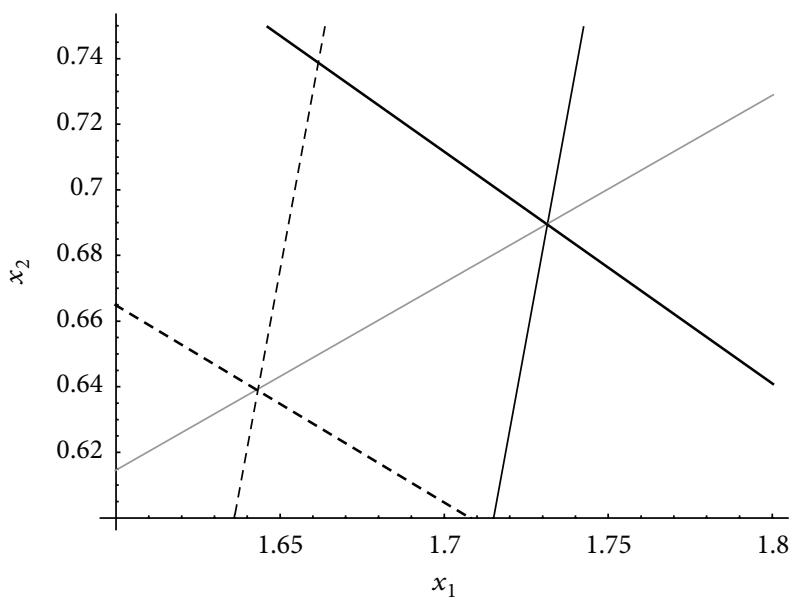

FIgURE 2: Zoom-in of the optimistic scheme, $f^{+}$.

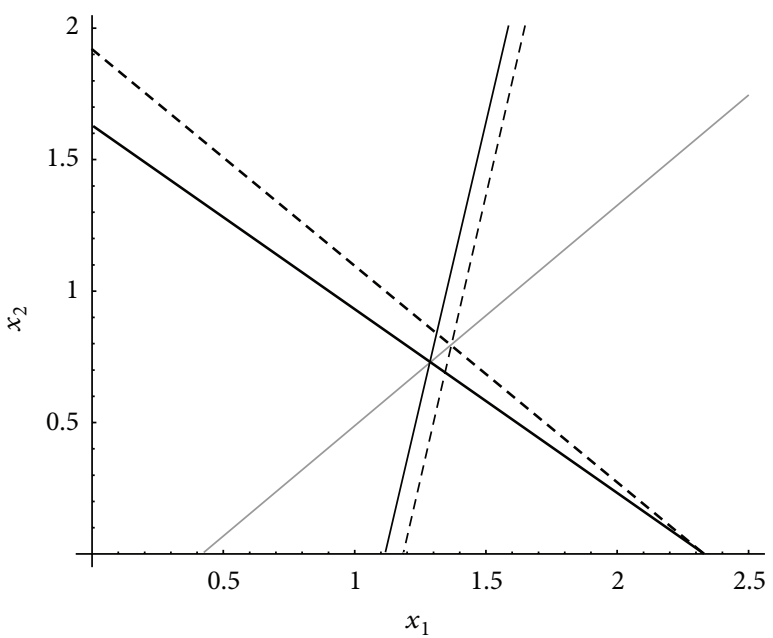

Figure 3: Conservative scheme, $f^{-}$.

In the second stage, substituting $x_{j}^{s}$ into the formula in (2) converts (2) into the following formula:

$$
\begin{array}{ll}
\operatorname{Max} & f^{ \pm}=\sum_{j=1}^{n}\left[c_{j}^{ \pm} x_{j}^{s}+d_{j}^{ \pm}\left(x_{j}^{s}\right)^{2}\right], \\
\text { s.t. } & \sum_{j=1}^{n} a_{i j}^{ \pm} x_{j}^{s} \leq b_{i}^{ \pm}, \quad i=1,2 \ldots, m, \\
& x_{j}^{s} \geq 0, \quad j=1,2, \ldots, n .
\end{array}
$$

To determine the coefficients $a_{i j}^{ \pm}, b_{i}^{ \pm}, c_{j}^{ \pm}$, and $d_{j}^{ \pm}$corresponding to $f^{ \pm}$we use the following:

$$
\begin{array}{ll}
\operatorname{Max} & f^{ \pm}=\sum_{j=1}^{n}\left[c_{j}^{ \pm} x_{j}^{s}+d_{j}^{ \pm}\left(x_{j}^{s}\right)^{2}\right], \\
\text { s.t. } & \sum_{j=1}^{n} a_{i j}^{ \pm} x_{j}^{s} \leq b_{i}^{ \pm}, \quad i=1,2, \ldots, m,
\end{array}
$$

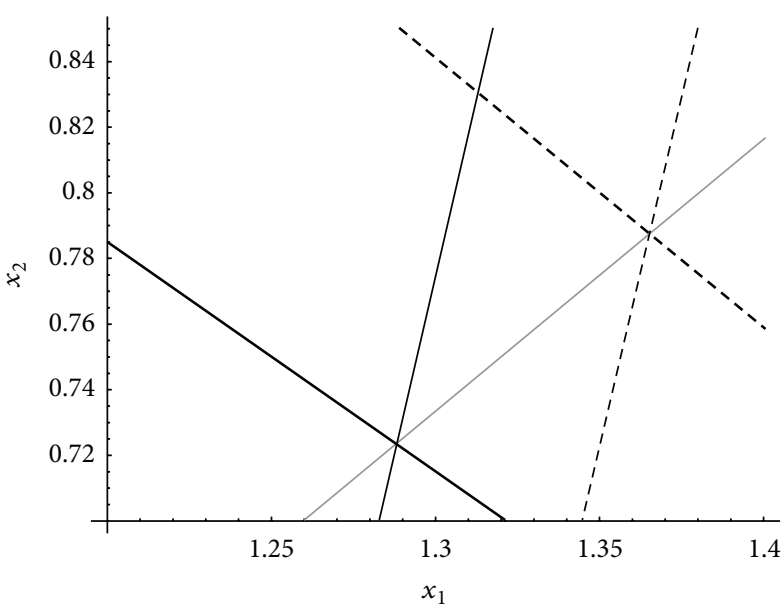

FIGURE 4: Zoom-in of the conservative scheme, $f^{-}$.

$$
\begin{array}{ll}
\text { Min } & f^{ \pm}=\sum_{j=1}^{n}\left[c_{j}^{ \pm} x_{j}^{s}+d_{j}^{ \pm}\left(x_{j}^{s}\right)^{2}\right], \\
\text { s.t. } & \sum_{j=1}^{n} a_{i j}^{ \pm} x_{j}^{s} \leq b_{i}^{ \pm}, \quad i=1,2, \ldots, m .
\end{array}
$$

To determine $a_{j}^{ \pm^{+}}$and $b_{i}^{ \pm^{+}}$of the optimistic scheme corresponding to the upper limit of the objective value of $f^{+}$, we have the following:

$$
\begin{array}{ll}
\operatorname{Max} & \sum_{j=1}^{n} \mathrm{abs}\left(a_{i j}^{ \pm} x_{j}^{s}-b_{i}^{ \pm}\right), \\
\text {s.t. } & \sum_{j=1}^{n} a_{i j}^{ \pm} x_{j}^{s} \leq b_{i}^{ \pm}, \quad i=1,2, \ldots, m .
\end{array}
$$

To obtain $a_{j}^{ \pm^{-}}$and $b_{i}^{ \pm-}$, we use the following:

$$
\begin{array}{ll}
\text { Min } & \sum_{j=1}^{n} a b s\left(a_{i j}^{ \pm} x_{j}^{s}-b_{i}^{ \pm}\right), \\
\text {s.t. } & \sum_{j=1}^{n} a_{i j}^{ \pm} x_{j}^{s} \leq b_{i}^{ \pm}, \quad i=1,2, \ldots, m .
\end{array}
$$

In the third stage, the problem expressed in (2) has been converted into the following two subproblems.

For $f^{+}$,

$$
\begin{array}{ll}
\operatorname{Max} & f^{+}=\sum_{j=1}^{n}\left[c_{j}^{ \pm+} x_{j}^{ \pm}+d_{j}^{ \pm+}\left(x_{j}^{ \pm}\right)^{2}\right], \\
\text { s.t. } & \sum_{j=1}^{n} a_{i j}^{ \pm+} x_{j}^{ \pm} \leq b_{i}^{ \pm+}, \quad i=1,2, \ldots, m, \\
& x_{j}^{ \pm} \geq 0, \quad j=1,2, \ldots, n .
\end{array}
$$


For $f^{-}$,

$$
\begin{array}{r}
\text { Max } \quad f^{-}=\sum_{j=1}^{n}\left[c_{j}^{ \pm-} x_{j}^{ \pm}+d_{j}^{ \pm^{-}}\left(x_{j}^{ \pm}\right)^{2}\right], \\
\text { s.t. } \quad \sum_{j=1}^{n} a_{i j}^{ \pm-} x_{j}^{ \pm} \leq b_{i}^{ \pm-}, \quad i=1,2, \ldots, m, \\
x_{j}^{ \pm} \geq 0, \quad j=1,2, \ldots, n .
\end{array}
$$

The inexact information has been incorporated in these two subproblems. These two subproblems, as typical nonlinear programming problems, can be solved by the GA nonlinear program solver engine of GASGOT.

This method is applied to an IQP problem that was originally proposed by [27]. This IQP problem can be expressed as follows:

$$
\begin{array}{cc}
\operatorname{Max} \quad f^{ \pm}= & {[16,18] x_{1}^{ \pm}-[12,14]\left(x_{1}^{ \pm}\right)^{2}} \\
& -[4,5] x_{2}^{ \pm}+[14,15]\left(x_{2}^{ \pm}\right)^{2}, \\
\text { s.t. } \quad[4.5,5.5] x_{1}^{ \pm}+[1.8,2.2] x_{2}^{ \pm} \leq[1.8,2.1], \\
& x_{1}^{ \pm}+[1.8,2.2] x_{2}^{ \pm} \leq[0.9,1.1], \\
& x_{1}^{ \pm} \geq 0, \quad x_{2}^{ \pm} \geq 0 .
\end{array}
$$

In stage one, suboptimal variables can be calculated using the GA nonlinear program solver engine of GASGOT:

$$
x_{1}^{s}=0.26497, \quad x_{2}^{s}=0.37772, \quad f^{s}=4.397
$$

In stage two, $x_{1}^{s}$ and $x_{2}^{s}$ are used to construct the objective functions expressed in (27), (28), (29), and (30) in order to determine the coefficients $a_{i j}^{ \pm^{+}}, b_{i}^{ \pm^{+}}, c_{j}^{ \pm^{+}}$, and $d_{j}^{ \pm^{+}}$and $a_{i j}^{ \pm^{-}}$, $b_{i}^{ \pm^{-}}, c_{j}^{ \pm^{-}}$, and ${d_{j}^{ \pm^{-}}}^{-}$. By solving (27), (28), (29), and (30), the coefficients are identified as follows:

$$
\begin{gathered}
a_{11}^{ \pm^{+}}=4.5, \quad a_{12}^{ \pm}=1.8, \quad a_{21}^{ \pm^{+}}=1.8, \\
b_{1}^{ \pm^{+}}=2.1, \quad{b_{2}^{ \pm+}}^{ \pm^{+}}=1.1, \\
c_{1}^{ \pm^{+}}=18, \quad c_{2}^{ \pm^{+}}=4, \quad d_{1}^{ \pm^{+}}=12, \quad d_{2}^{ \pm^{+}}=15, \\
a_{11}^{ \pm^{-}}=5.5, \quad a_{12}^{ \pm-}=2.2, \quad a_{21}^{ \pm^{-}}=2.2, \\
b_{1}^{ \pm^{-}}=1.8, \quad b_{2}^{ \pm^{-}}=0.9, \\
c_{1}^{ \pm^{-}}=16, \quad c_{2}^{ \pm^{-}}=5, \quad d_{1}^{ \pm^{-}}=14, \quad d_{2}^{ \pm^{-}}=14 .
\end{gathered}
$$

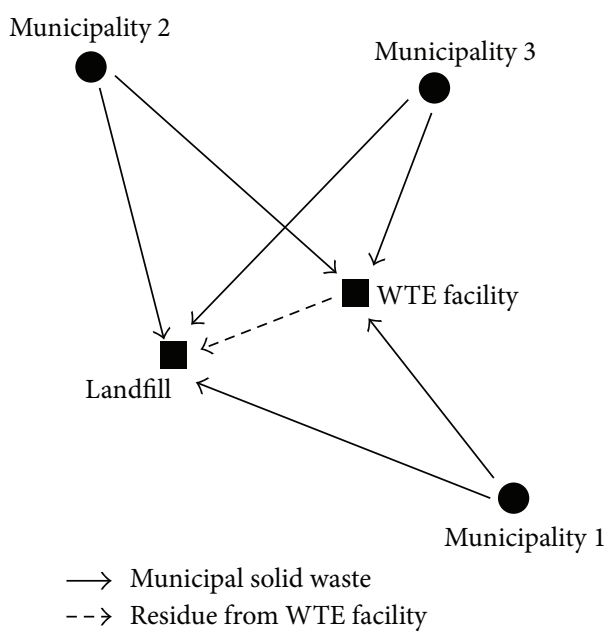

FIGURE 5: Diagram of municipalities and waste management facilities in the case study.

In stage three, the problems of (31) and (32) are generated as follows:

$$
\begin{array}{ll}
\text { Max } & f^{+}=18 x_{1}^{ \pm}-12\left(x_{1}^{ \pm}\right)^{2}-4 x_{2}^{ \pm}+15\left(x_{2}^{ \pm}\right)^{2}, \\
\text { s.t. } & 4.5 x_{1}^{ \pm}+1.8 x_{2}^{ \pm} \leq 2.1, \\
& x_{1}^{ \pm}+1.8 x_{2}^{ \pm} \leq 1.1, \\
& x_{1}^{ \pm} \geq 0, \quad x_{2}^{ \pm} \geq 0, \\
\text { Max } & f^{-}=16 x_{1}^{ \pm}-14\left(x_{1}^{ \pm}\right)^{2}-5 x_{2}^{ \pm}+14\left(x_{2}^{ \pm}\right)^{2}, \\
\text { s.t. } & 5.5 x_{1}^{ \pm}+2.2 x_{2}^{ \pm} \leq 1.8, \\
& x_{1}^{ \pm}+2.2 x_{2}^{ \pm} \leq 0.9, \\
& x_{1}^{ \pm} \geq 0, \quad x_{2}^{ \pm} \geq 0 .
\end{array}
$$

Solving the above two problems, the solution of this sample problem is $f^{ \pm}=[2.6371,5.4234], x_{1}^{ \pm}=[0.2441,0.2857]$, and $x_{2}^{ \pm}=[0.20792,0.45239]$. As a comparison, the solution given by [27] is $f^{ \pm}=[2.59,5.42], x_{1}^{ \pm}=[0.238,0.286]$, and $x_{2}^{ \pm}=$ $[0.224,0.452]$.

To enhance the solution, the GAIQP engine can be reconfigured in stage three. For example, the maximum genetics generations number can be increased. However, this may not be necessary as the above generated solution is sufficiently satisfactory for many practical engineering problems.

\section{Case Study}

To illustrate the proposed method, the problem of solid waste disposal planning presented in [25] has been recalculated using the GAIQP method. In this case study, the system involves three cities. As shown in Figure 5, the planning horizon is 15 years, which is divided equally into three periods. A landfill and an incinerator are available for the disposal of the municipal solid waste. The landfill has an 
existing capacity of $[2.05,2.30] \times 10^{6} \mathrm{t}$, and the incinerator has a capacity of $[500,600] \mathrm{t} / \mathrm{d}$. The incinerator generates residues of approximately $30 \%$ of the incoming waste streams, and its revenue from energy sale is $\$[15,25]$ per ton combusted. The waste generation rate of each city, operating costs of the facilities, and the waste transportation costs are summarized and shown in Table 2.

The objective of the optimization problem in this case study is to minimize the total costs by allocating waste flow between cities and facilities. In [25], this IQP model was formulated as follows:

$$
\begin{aligned}
& \operatorname{Min} f^{ \pm}=\sum_{i=1}^{2} \sum_{j=1}^{3} \sum_{k=1}^{3} L_{k}\left(T R_{i j k}^{ \pm}+O P_{i k}^{ \pm}\right) x_{i j k}^{ \pm} \\
& +\sum_{j=1}^{3} \sum_{k=1}^{3} L_{k}\left[F E\left(F T_{k}^{ \pm}+O P_{2 k}^{ \pm}\right)-R E_{k}^{ \pm}\right] x_{2 j k}^{ \pm} \\
& \text {(where } T R_{i j k}^{ \pm}=\alpha_{i j k}^{ \pm} x_{i j k}^{ \pm}+\beta_{i j k}^{ \pm} \text {, } \\
& \left.F T_{j k}^{ \pm}=\sigma_{k}^{ \pm} x_{2 j k}^{ \pm} F E+\delta_{k}^{ \pm}\right), \\
& \text {s.t. } \quad \sum_{j=1}^{3} \sum_{k=1}^{3} L_{k}\left(x_{1 j k}^{ \pm}+F E x_{2 j k}^{ \pm}\right) \\
& \leq T L^{ \pm} \quad \text { (landfill capacity constraint) }, \\
& \sum_{j=1}^{3} x_{2 j k}^{ \pm} \leq T E^{ \pm} \quad \forall k \text { (incinerator } \\
& \text { capacity constraint), } \\
& \sum_{i=1}^{2} x_{i j k}^{ \pm} \geq W G_{j k}^{ \pm} \quad \forall j, k \text { (waste disposal } \\
& \text { demand constraint), } \\
& x_{i j k}^{ \pm} \geq 0 \quad \forall i, j, k \text { (nonnegativity constraint), }
\end{aligned}
$$

where

$F E$ is residue flow rate from incinerator to landfill (it is 0.3 in this case),

$F T_{k}^{ \pm}$is transportation cost for residue from incinerator to landfill during period $k(\$ / \mathrm{t})$,

$i$ is type of waste management facility $(i=1,2$, where $i=1$ for landfill, 2 for incinerator),

$j$ is city, $j=1,2,3$,

$k$ is time period, $k=1,2,3$,

$L_{k}$ is length of period $k, L_{1}=L_{2}=L_{3}=365 * 5$ (day), $O P_{i k}^{ \pm}$is operating cost of facility $i$ during period $k(\$ / t)$,

$R E_{k}^{ \pm}$is revenue from incinerator during period $k(\$ / t)$, $R E_{1}^{ \pm}=R E_{2}^{ \pm}=R E_{3}^{ \pm}=[15,25]$,

$T E^{ \pm}$is capacity of incinerator $(\mathrm{t} / \mathrm{d})$,
$T L^{ \pm}$is capacity of landfill $(\mathrm{t})$,

$T L_{i j k}^{ \pm}$is transportation cost for waste from city $j$ to facility $i$ during period $k(\$ / t)$,

$W G_{j k}^{ \pm}$is waste generation rate in city $j$ during period $k(\mathrm{t} / \mathrm{d})$,

$x_{i j k}^{ \pm}$is waste flow from city $j$ to facility $i$ during period $k(\mathrm{t} / \mathrm{d})$,

$\alpha_{i j k}^{ \pm}$is slope of transportation cost curve for waste from city $j$ to facility $i$, during period $k$,

$\beta_{i j k}^{ \pm}$is $Y$-intercept of transportation cost curve for waste from city $j$ to facility $i$ during period $k$,

$\delta_{k}^{ \pm}$is $Y$-intercept of transportation cost curve for residue from incinerator to landfill during period $k$,

$\sigma_{k}^{ \pm}$is Slope of transportation cost curve for residue from incinerator to landfill during period $k$.

This interval quadratic programming problem has 18 variables $x_{i j k}$ and 13 constraints. The GAIQP method is applied to solve this problem according to the three stages presented in Section 3.2.

Stage 1. Based on the data shown in Table 2, the model formulated at stage 1 is as follows:

$$
\min f=365 \times 5
$$

$$
\times\left\{[44.6,64.4] x_{111}\right.
$$$$
-[0.0123,0.0163] x_{111}^{2}
$$$$
+[56.04,81.34] x_{112}
$$$$
-[0.0135,0.0179] x_{112}^{2}
$$$$
+[67.64,103.48] x_{113}
$$$$
-[0.0148,0.0197] x_{113}^{2}
$$$$
+[42.65,61.9] x_{121}
$$$$
-[0.0106,0.0142] x_{121}^{2}
$$$$
+[53.9,78.56] x_{122}
$$$$
-[0.0117,0.0156] x_{122}^{2}
$$ 
TABLE 2: Waste generation rates and operating costs.

\begin{tabular}{|c|c|c|c|}
\hline & Period $1(k=1)$ & Period $2(k=2)$ & Period $3(k=3)$ \\
\hline \multicolumn{4}{|c|}{ Waste generation $(\mathrm{t} / \mathrm{d})$} \\
\hline $\mathrm{WG}_{1 k}^{ \pm}$ & {$[260,340]$} & {$[310,390]$} & {$[360,440]$} \\
\hline $\mathrm{WG}_{2 k}^{ \pm}$ & {$[160,240]$} & {$[185,265]$} & {$[210,290]$} \\
\hline $\mathrm{WG}_{3 k}^{ \pm}$ & {$[260,340]$} & {$[260,340]$} & {$[310,390]$} \\
\hline \multicolumn{4}{|c|}{ Operation cost $(\$ / t)$} \\
\hline $\mathrm{OP}_{1 k}^{ \pm}$ & {$[35,45]$} & {$[40,60]$} & {$[50,80]$} \\
\hline $\mathrm{OP}_{2 k}^{ \pm}$ & {$[55,75]$} & {$[60,85]$} & {$[65,95]$} \\
\hline \multicolumn{4}{|c|}{ City-to-landfill transportation cost $(\$ / \mathrm{t})$} \\
\hline $\mathrm{TR}_{11 k}^{-}$ & $-0.0123 x+14.58$ & $-0.0135 x+16.04$ & $-0.0148 x+17.64$ \\
\hline $\mathrm{TR}_{11 k}^{+}$ & $-0.0163 x+19.40$ & $-0.0179 x+21.34$ & $-0.0197 x+23.48$ \\
\hline $\mathrm{TR}_{12 k}^{-}$ & $-0.0106 x+12.65$ & $-0.0117 x+13.92$ & $-0.0129 x+15.31$ \\
\hline $\mathrm{TR}_{12 k}^{+}$ & $-0.0142 x+16.87$ & $-0.0156 x+18.56$ & $-0.0172 x+20.41$ \\
\hline $\mathrm{TR}_{13 k}^{-}$ & $-0.0129 x+15.30$ & $-0.0141 x+16.83$ & $-0.0156 x+18.52$ \\
\hline $\mathrm{TR}_{13 k}^{+}$ & $-0.0172 x+20.49$ & $-0.0189 x+22.53$ & $-0.0208 x+24.79$ \\
\hline \multicolumn{4}{|c|}{ City-to-incinerator transportation cost $(\$ / t)$} \\
\hline $\mathrm{TR}_{21 k}^{-}$ & $-0.0097 x+11.57$ & $-0.0107 x+12.73$ & $-0.0118 x+14.00$ \\
\hline $\mathrm{TR}_{21 k}^{+}$ & $-0.0130 x+15.42$ & $-0.0143 x+16 / 97$ & $-0.0157 x+18.66$ \\
\hline $\mathrm{TR}_{22 k}^{-}$ & $-0.0102 x+12.17$ & $-0.0113 x+13.39$ & $-0.0124 x+14.73$ \\
\hline $\mathrm{TR}_{22 k}^{+}$ & $-0.0136 x+16.15$ & $-0.0149 x+17.76$ & $-0.0164 x+19.54$ \\
\hline $\mathrm{TR}_{23 k}^{-}$ & $-0.0089 x+10.60$ & $-0.0098 x+11.67$ & $-0.0108 x+12.83$ \\
\hline $\mathrm{TR}_{23 k}^{+}$ & $-0.0118 x+14.10$ & $-0.0130 x+15.51$ & $-0.0143 x+17.06$ \\
\hline \multicolumn{4}{|c|}{ Incinerator-to-landfill transportation cost $(\$ / \mathrm{t})$} \\
\hline $\mathrm{FT}_{k}^{-}$ & $-0.0048 x+5.71$ & $-0.0053 x+6.28$ & $-0.0058 x+6.91$ \\
\hline $\mathrm{FT}_{k}^{+}$ & $-0.0064 x+7.62$ & $-0.0070 x+8.38$ & $-0.0077 x+9.33$ \\
\hline
\end{tabular}

$$
\begin{aligned}
& +[65.3,100.4] x_{123} \\
& -[0.0129,0.0172] x_{123}^{2} \\
& +[45.3,65.5] x_{131} \\
& -[0.0129,0.0172] x_{131}^{2} \\
& +[56.83,82.53] x_{132} \\
& -[0.0141,0.0189] x_{132}^{2} \\
& +[58.52,104.79] x_{133} \\
& -[0.0156,0.0208] x_{133}^{2} \\
& +[60.6,100.4] x_{211} \\
& -[0.0111,0.015] x_{211}^{2}+[64.2,115.3] x_{212} \\
& -[0.0122,0.0164] x_{212}^{2} \\
& +[74.4,126.73] x_{213} \\
& -[0.0134,0.018] x_{213}^{2} \\
& +[54.3,103.44] x_{221} \\
& -[0.0117,0.0157] x_{221}^{2} \\
& +
\end{aligned}
$$

s.t. $365 \times 5$

$$
\begin{aligned}
& \times\left(x_{111}+0.3 x_{211}+x_{112}+0.3 x_{212}\right. \\
& +x_{113}+0.3 x_{213}+x_{121}+0.3 x_{221} \\
& +x_{122}+0.3 x_{222}+x_{123}+0.3 x_{223} \\
& \quad+x_{131}+0.3 x_{231}+x_{132}+0.3 x_{232} \\
& \left.+x_{123}+0.3 x_{233}\right)
\end{aligned}
$$




$$
\begin{gathered}
\leq\left[2.05 \times 10^{6}, 2.30 \times 10^{6}\right], \\
x_{211}+x_{221}+x_{231} \leq[500,600], \\
x_{212}+x_{222}+x_{232} \leq[500,600], \\
x_{213}+x_{223}+x_{233} \leq[500,600], \\
x_{111}+x_{211} \geq[260,340], \\
x_{112}+x_{212} \geq[310,390], \\
x_{113}+x_{213} \geq[360,440], \\
x_{121}+x_{221} \geq[160,240], \\
x_{122}+x_{222} \geq[185,265], \\
x_{131}+x_{231} \geq[260,340], \\
x_{132}+x_{232} \geq[260,340], \\
x_{133}+x_{233} \geq[310,390] .
\end{gathered}
$$

Considering interval numbers as random numbers, which can be determined between the interval numbers' lower and upper endpoints, the GAIQP method is applied to find a suboptimal solution $f^{s}$. With an initial population size of 500 , after 2000 generations, one suboptimal solution has been found: $f^{s}=288 \times 10^{6}$, and the concomitant variables are

$$
\begin{array}{cll}
x_{111}^{s}=250, & x_{112}^{s}=315, & x_{113}^{s}=376, \\
x_{121}^{s}=3, & x_{122}^{s}=220, & x_{123}^{s}=57, \\
x_{131}^{s}=8, & x_{132}^{s}=10, & x_{133}^{s}=5, \\
x_{211}^{s}=45, & x_{212}^{s}=40, & x_{213}^{s}=10, \\
x_{221}^{s}=188, & x_{222}^{s}=12, & x_{223}^{s}=201, \\
x_{231}^{s}=278, & x_{232}^{s}=293, & x_{233}^{s}=322 .
\end{array}
$$

Stage 2. Substitute $x_{i j k}^{s}$ into (38) to identify those coefficients corresponding to $f^{+}$and $f^{-}$.

Stage 3. In this stage, the problem expressed in (37) has been converted into two subproblems: $f^{+}$and $f^{-}$.

The solution found by this method is $f^{ \pm}=[2.405 \times$ $\left.10^{8}, 5.133 \times 10^{8}\right]$. This is close to the result given by [25], which is, for comparison purposes, $f^{ \pm}=\left[2.39 \times 10^{8}, 5.14 \times 10^{8}\right]$. This case study indicates that the GAIQP method can be configured to deal with large-scaled and complex engineering problems and give a satisfactory solution. If the parameters of the genetic algorithms of the nonlinear program solver engine of GASGOT are further tuned, a better solution can be generated.

\section{Conclusions}

Two genetic-algorithms-based methods have been proposed and applied for identifying an all-purpose optimization solution for the inexact linear programming and Inexact quadratic programming problems. The two methods are called GAILP and GAIQP. The Genetic Algorithm Solver of MATLAB was the implementation environment of the proposed methods. Compared to the GAILP and GAIQP methods, the traditional problem-solving method has limitations due to the complexity involved in selecting the upper or lower bounds of variables and parameters when the subobjective functions are being constructed. The complexity arises due to the extensive computation and necessary assumptions and simplification. The solution procedures of the proposed GA-based optimization methods do not involve any such assumption or simplification, and the quality of the result is guaranteed. The GAIQP method has been applied to a case study that deals with municipal solid waste management taken from [25]. A comparison of the results shows that the proposed GA-based heuristic optimization approach is able to handle more complicated quadratic relationships involving uncertainty and provide better results.

The GA-based heuristic optimization approach is a flexible approach, which can be extended to find solutions for various types of operation programming scenarios. It can also be used as an all-purpose algorithm for economic optimizations. In the future, the methods of GAILP and GAIOP will be further developed for handling more complex inexact nonlinear problems.

\section{Acknowledgment}

The financial support of the Canada Research Chair Program of Canada is gratefully acknowledged.

\section{References}

[1] L. E. Anderson, "A mathematical model for the optimization of a waste management system," Technical Report 68-1, Sanitary Engineering Research Laboratory, University of California, Berkely, Calif, USA, 1968.

[2] H. L. Christensen and G. F. Haddix, "A model for sanitary landfill management and design," Computers and Operations Research, vol. 1, no. 2, pp. 275-281, 1974.

[3] L. A. Fuertes, J. F. Hundson, and D. H. Mark, "Solid Waste Management: equity trade-off models," Journal of Urban Planning and Development, vol. 100, pp. 155-171, 1974.

[4] T. L. Jacobs and J. W. Everett, "Optimal scheduling of consecutive landfill operations with recycling," Journal of Environmental Engineering, vol. 118, no. 3, pp. 420-429, 1992.

[5] L. Jenkins, "Parametric mixed integer programming - an application to solid waste management," Management Science, vol. 28, no. 11, pp. 1270-1284, 1982.

[6] N.-B. Chang, C. G. Wen, Y. L. Chen, and Y. C. Yong, "A grey fuzzy multiobjective programming approach for the optimal planning of a reservoir watershed. Part B: application," Water Research, vol. 30, no. 10, pp. 2335-2340, 1996.

[7] N.-B. Chang, C. A. Shoemaker, and R. E. Schuler, "Solid waste management system analysis with air pollution and leachate impact limitations," Waste Management and Research, vol. 14, no. 5, pp. 463-481, 1996.

[8] S. Sushil and P. Vrat, "Waste management policy analysis and growth monitoring: an integrated approach to perspective 
planning," International Journal of Systems Science, vol. 20, no. 6, pp. 907-926, 1989.

[9] N.-B. Chang, C. G. Wen, Y. L. Chen, and Y. C. Yong, "A grey fuzzy multiobjective programming approach for the optimal planning of a reservoir watershed. Part A: theoretical development," Water Research, vol. 30, no. 10, pp. 2329-2334, 1996.

[10] M. Ekmekçioĝlu, T. Kaya, and C. Kahraman, "Fuzzy multicriteria disposal method and site selection for municipal solid waste," Waste Management, vol. 30, no. 8-9, pp. 1729-1736, 2010.

[11] Y. Li and G. Huang, "Dynamic analysis for solid waste management systems: an inexact multistage integer programming approach," Journal of the Air and Waste Management Association, vol. 59, no. 3, pp. 279-292, 2009.

[12] Y. M. Zhang, G. H. Huang, and L. He, "An inexact reverse logistics model for municipal solid waste management systems," Journal of Environmental Management, vol. 92, no. 3, pp. 522530, 2011.

[13] N.-B. Chang and S. F. Wang, "A fuzzy goal programming approach for the optimal planning of metropolitan solid waste management systems," European Journal of Operational Research, vol. 99, no. 2, pp. 303-321, 1997.

[14] P. Guo and G. H. Huang, "Inexact fuzzy-stochastic mixedinteger programming approach for long-term planning of waste management-Part A: methodology," Journal of Environmental Management, vol. 91, no. 2, pp. 461-470, 2009.

[15] Q. Tan, G. H. Huang, and Y. Cai, "A superiority-inferioritybased inexact fuzzy stochastic programming approach for solid waste management under uncertainty," Environmental Modeling and Assessment, vol. 15, no. 5, pp. 381-396, 2010.

[16] Y. W. Lee, I. Bogardi, and J. Stansbury, "Fuzzy decision making in dredged-material management," Journal of Environmental Engineering, vol. 117, no. 5, pp. 614-630, 1991.

[17] Y. Sun, Y. Li, and G. Huang, "Development of a fuzzy-queuebased interval linear programming model for municipal solid waste management," Environmental Engineering Science, vol. 27, no. 6, pp. 451-468, 2010.

[18] G. Huang, B. W. Baetz, and G. G. Patry, "Grey quadratic programming and its application to municipal solid waste management planning under uncertainty," Engineering Optimization, vol. 23, pp. 201-223, 1994.

[19] G. H. Huang, B. W. Baetz, and G. G. Patry, "Grey integer programming: an application to waste management planning under uncertainty," European Journal of Operational Research, vol. 83, no. 3, pp. 594-620, 1995.

[20] G. Huang and R. D. Moore, "Grey linear programming, its solving approach, and its application," International Journal of Systems Science, vol. 24, no. 1, pp. 159-172, 1993.

[21] G. H. Huang, B. W. Baetz, and G. G. Patry, "Grey dynamic programming for waste-management planning under uncertainty," Journal of Urban Planning \& Development, vol. 120, no. 3, pp. 132-156, 1994.

[22] G. H. Huang, B. W. Baetz, and G. G. Patry, "Grey dynamic programming for waste-management planning under uncertainty," Journal of Urban Planning \& Development, vol. 120, no. 3, pp. 132-156, 1994.

[23] G. Huang, B. W. Baetz, and G. G. Patry, "A grey fuzzy linear programming approach for municipal solid waste management planning under uncertainty," Civil Engineering Systems, vol. 10, no. 2, pp. 123-146, 1993.

[24] G. Huang, B. W. Baetz, and G. G. Patry, "Waste flow allocation planning through a grey fuzzy quadratic programming approach," Civil Engineering Systems, vol. 11, pp. 209-243, 1994.
[25] G. H. Huang, B. W. Baetz, and G. G. Patry, "Grey fuzzy integer programming: an application to regional waste management planning under uncertainty," Socio-Economic Planning Sciences, vol. 29, no. 1, pp. 17-38, 1995.

[26] G. Huang, B. W. Baetz, and G. G. Patry, "A grey linear programming approach for municipal solid waste management planning under uncertainty," Civil Engineering Systems, vol. 9, no. 4, pp. 319-335, 1992.

[27] M. J. Chen and G. H. Huang, "A derivative algorithm for inexact quadratic program-application to environmental decisionmaking under uncertainty," European Journal of Operational Research, vol. 128, no. 3, pp. 570-586, 2001. 


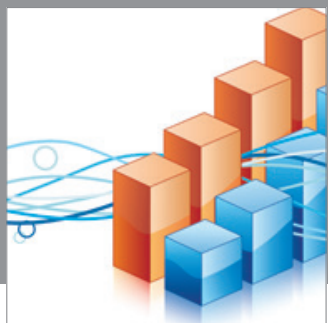

Advances in

Operations Research

mansans

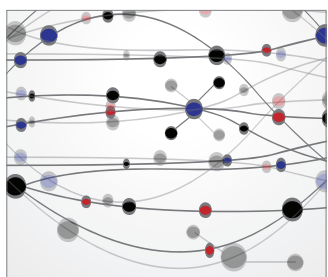

The Scientific World Journal
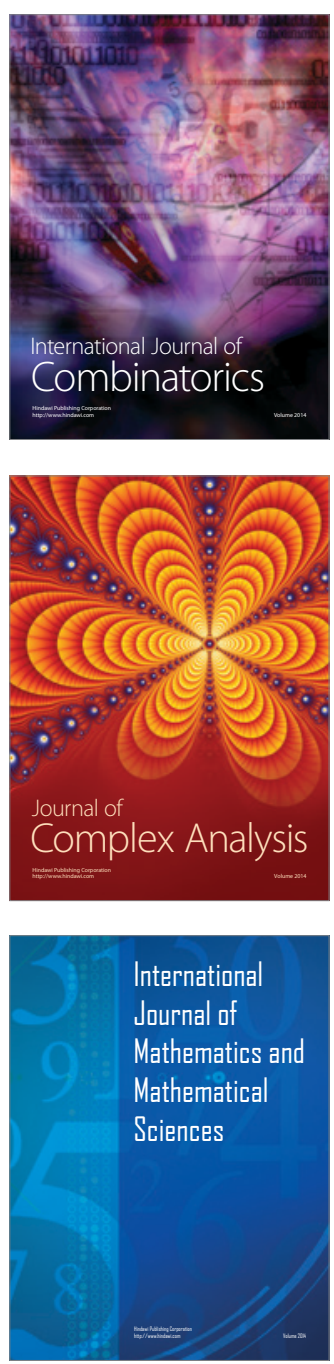
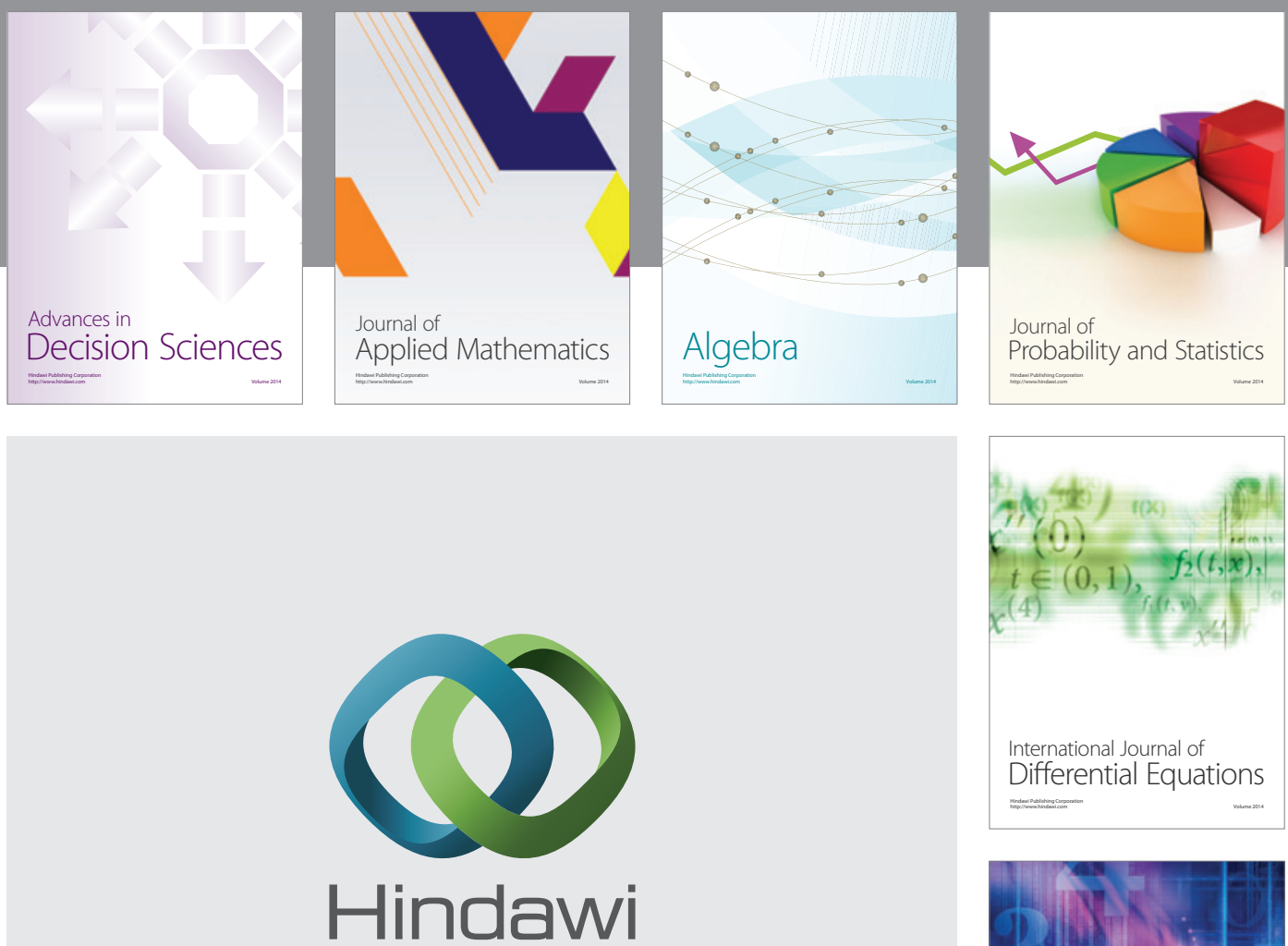

Submit your manuscripts at http://www.hindawi.com
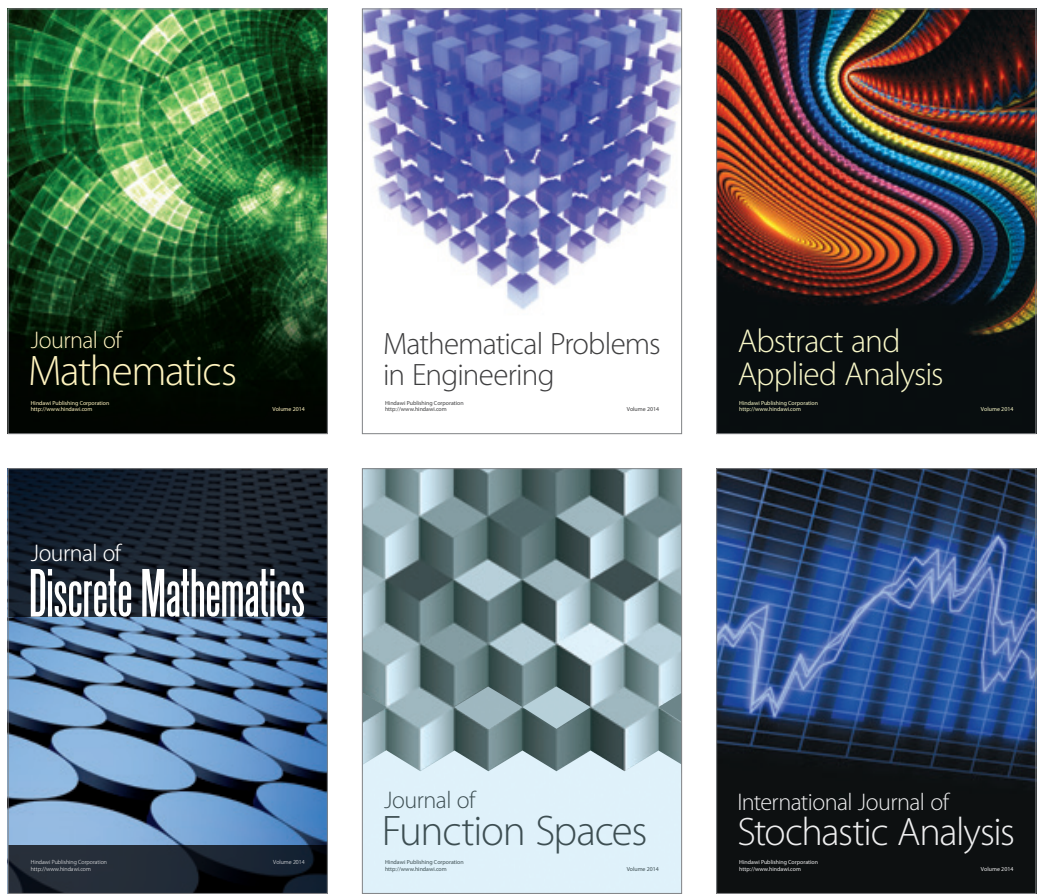

Journal of

Function Spaces

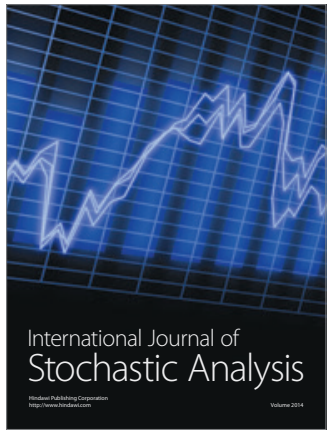

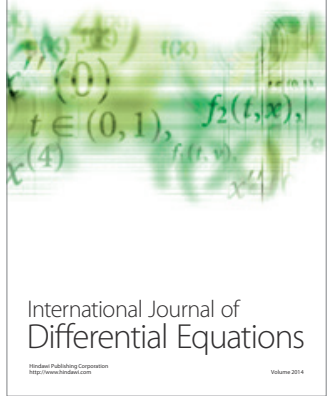
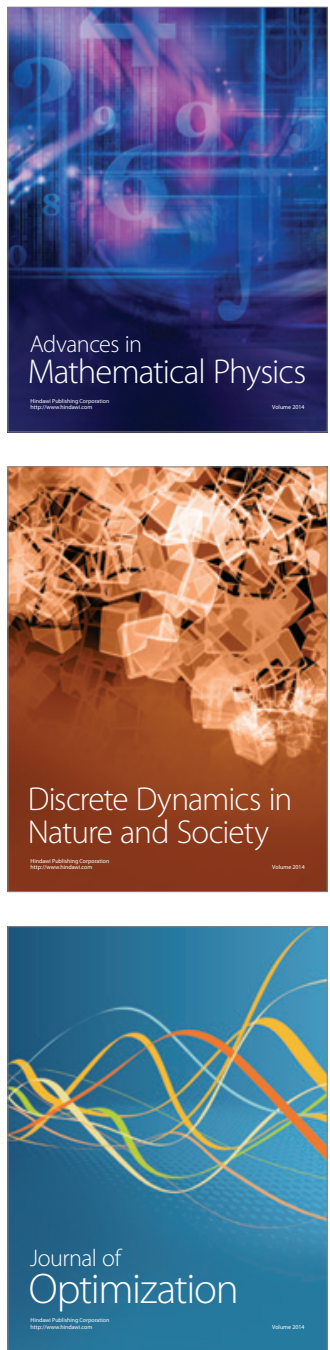